\title{
Construção de uma tecnologia assistencial para auxílio a pacientes com prejuízo vocal pós-cirúrgico: relato de experiência
}

\author{
Construction of an assistive technology to help patients with post-surgical vocal \\ impairment: an experience report
}
Construcción de una tecnología de asistencia para ayudar a los pacientes con discapacidad vocal posquirúrgica: un informe de experiencia

Amanda Rodrigues Figueiredo ${ }^{*}$, Ana Beatriz Souza Cabral ${ }^{1}$, Douglas Rafael da Cruz Carneiro ${ }^{1}$, Jorgeany Soares Parente ${ }^{1}$, Márcio Alves Ribeiro'.

\section{RESUMO}

Objetivo: Relatar a experiência acadêmica em um programa de iniciação científica sobre a construção de uma tecnologia assistencial para o auxílio à comunicação não verbal de pacientes em pós-operatório por cânceres de cabeça e pescoço com prejuízo vocal. Relato de experiência: A tecnologia foi desenvolvida por acadêmicos do $10^{\circ}$ semestre do curso de bacharelado em enfermagem, como produto ligado ao Programa de Iniciação Científica. O instrumento foi desenvolvido, em forma de cubo, contendo em cada uma de suas seis faces uma frase curta ou palavra associada a uma imagem e cor diferentes. Antes da construção física do instrumento, foi elaborado um esboço de arte gráfica com o conteúdo de cada face do cubo. As etapas de montagem dos cubos foram realizadas pelos discentes integrantes do projeto de pesquisa. Considerações finais: A participação em um programa de iniciação científica, a execução de um estudo metodológico, culminando com a construção de uma tecnologia leve-dura de caráter assistivo favorecem a formação dos acadêmicos.

Palavras-chave: Câncer de cabeça e pescoço, Comunicação não verbal, Tecnologia, Enfermagem.

\begin{abstract}
Objective: To report the academic experience in a scientific initiation program on the construction of an assistive technology to aid non-verbal communication in postoperative patients for head and neck cancers with vocal impairment. Experience report: The technology was developed by students of the 10th semester of the Bachelor's Degree in Nursing, as a product linked to the Scientific Initiation Program. The instrument was developed in the form of a cube, containing on each of its six sides a short phrase or word associated with a different image and color. Before the physical construction of the instrument, a graphic art sketch was elaborated with the contents of each face of the cube. The steps of assembling the cubes were carried out by the students participating in the research project. Final considerations: Participating in a scientific initiation program, carrying out a methodological study, culminating in the construction of a light-hard assistive technology, favor the training of students.
\end{abstract}

Key words: Head and neck cancer, Nonverbal communication, Technology, Nursing.

\section{RESUMEN}

Objetivo: Informar la experiencia académica en un programa de iniciación científica sobre la construcción de una tecnología asistencial para ayudar a la comunicación no verbal en pacientes posoperatorios por cánceres

${ }^{1}$ Centro Universitário Metropolitano da Amazônia (UNIFAMAZ), Belém - PA.

*E-mail: amandar.figueiredo29@gmail.com

SUBMETIDO EM: 11/2021

PUBLICADO EM: 1/2022 
de cabeza y cuello con deterioro vocal. Informe de experiencia: La tecnología fue desarrollada por estudiantes del décimo semestre de la Licenciatura en Enfermería, como un producto vinculado al Programa de Iniciación Científica. El instrumento fue desarrollado en forma de cubo, conteniendo en cada uno de sus seis lados una frase corta o palabra asociada a una imagen y color diferente. Antes de la construcción física del instrumento, se elaboró un boceto de arte gráfico con el contenido de cada cara del cubo. Los pasos de montaje de los cubos fueron realizados por los estudiantes que participaron en el proyecto de investigación. Consideraciones finales: Participar en un programa de iniciación científica, realizar un estudio metodológico, que culmine en la construcción de una tecnología asistencial ligera-dura, favorezca la formación de los estudiantes.

Palabras clave: Cáncer de cabeza y cuello, Comunicación no verbal, Tecnología, Enfermería.

\section{INTRODUÇÃO}

O Câncer de Cabeça e Pescoço (CCP) configura-se com mais frequência com o carcinoma espinocelular apresentando-se em cerca de $90 \%$ das ocorrências. O tumor af eta as regiões do trato digestivo superior que inclui a cavidade oral com $40 \%$ dos casos, principalmente na mucosa da boca, língua, lábios, assoalho bucal e palato duro; $15 \%$ ocorrem na faringe, neste caso incluem-se a hipofaringe, orofaringe e nasofaringe; e $25 \%$ na laringe, surgem menores ocorrências na tireoide e glândulas salivares (NEVES CPB, et al., 2017).

A estimativa para o triênio de 2020-2022 no Brasil é de 625 mil novos casos. Neste contexto, a distribuição da ocorrência por região geográfica tende a apresentar variações de magnitude e nos tipos de cânceres entre as diferentes áreas geográficas brasileiras (INSTITUTO NACIONAL DO CÂNCER (INCA), 2019).

O CCP apresenta fatores de riscos tanto genéticos como ambientais que são contribuintes para sua etiologia, tal como o consumo excessivo de álcool e tabaco, que estão diretamente relacionados com o acometimento do trato aerodigestivo superior que inclui as regiões da cavidade oral, faringe, laringe e tireoide (SILVA FA, et al., 2020).

O tratamento cirúrgico para o CCP pode levar a prejuízos na qualidade de vida, já que podem ser desfigurante e psicologicamente traumático e manifestar alterações como prejuízos funcionais na mastigação e deglutição e alterações vocais (RUFINO PJH, 2020).

Outras consequências físicas e funcionais relacionadas a cirúrgica do CCP incluem disfagia, odinofagia, xerostomia, dor, trismo, mobilidade limitada da língua, abertura oral limitada, perda da estrutura mandibular ou maxilar, condições que geralmente af etam a fala (BARROS ES, 2017).

Para tanto, construção de uma metodologia de comunicação baseada em tecnologia leve-dura de certo vem a favorecer o cuidado a esse público, otimizando a comunicação, o que é fundamental para a manutenção dos processos de enfermagem frente a atenção ao paciente afônico em pós-operatónio (ALCANTARA LS, 2017).

As tecnologias assistivas são dispositivos que contribuem para o processo do cuidar. Esse tipo de tecnologia é extremamente importante para auxiliar na promoção de uma assistência integral baseada nas particularidades de cada paciente. Além disso é um instrumento de consolidação do plano de cuidados das equipes de saúde, de protocolos e da padronização de procedimentos (SILVA IR, et al., 2017).

Métodos de comunicação não verbais, baseadas em tecnologias leves e leves-duras, demonstram ser a melhor alternativa para comunicação com pacientes afônicos em pós-operatórios por tumores de cabeça e pescoço, pois são recursos acessíveis, simples e menos dispendiosos, prezando pela praticidade, visto que, tendem a se encaixar ao uso cotidiano (CAÇADOR MCRC, 2019).

A aplicação de uma tecnologia assistencial se mostra relevante para o desenvolvimento das atividades do cuidado de enfermagem, pois se torna meio para que o profissional auxilie o paciente a relatar seus problemas e necessidades básicas, sua participação na relação com o paciente e, também encontrar novas posturas de acordo com a situação vivenciada (RODRIGUES JCQ, 2019). 
A enfermagem busca aprimorar novos métodos destinados ao cuidado aos pacientes com função vocal comprometida no pós-operatório de CCP, pois a percepção e intervenção sobre sintomas como dor, ansiedade, estresse e outros é entendida como parte de uma abordagem assistencial diferenciada (NOGUEIRA CSF, 2017).

Neste sentido, a aplicabilidade de tecnologias assistenciais nas práticas interlocutoras com pacientes póscirúrgicos é favorável para interação entre cuidador e cuidado, pois a comunicação é uma atividade básica das relações interpessoais e preponderante nas ações junto aos pacientes, fundamental na inclusão, na empatia e na humanização (ALMEIDA JF, 2021).

Frente aos diversos aspectos sobre o câncer de cabeça e pescoço e considerando a necessidade de elaborar uma tecnologia assistencial capaz de responder aos anseios dos pacientes, questionou-se: Qual a experiência acadêmica em iniciação cientifica para a construção de uma tecnologia assistencial para auxílio na comunicação não verbal de pacientes em pós-operatório por cânceres de cabeça e pescoço com prejuízo vocal? Dessa forma o objetivo deste estudo foi relatar a experiência acadêmica em um programa de iniciação cientifica voltado à construção de uma tecnologia assistencial para o auxílio à comunicação não verbal de pacientes em pós-operatório por cânceres de cabeça e pescoço com prejuízo vocal.

\section{RELATO DE EXPERIÊNCIA}

Tratou-se de um estudo de abordagem qualitativa, descritivo, do tipo relato de experiência sobre a construção de uma tecnologia assistencial para ser aplicada na comunicação não verbal de pacientes em pós-operatório por câncer de cabeça e pescoço, cuja função vocal está comprometida. A tecnologia foi desenvolvida por acadêmicos do $10^{\circ}$ semestre do curso de bacharelado em enfermagem de um Centro Universitário no Estado do Pará como produto ligado ao Programa de Iniciação Científica vinculado à referida instituição, no período de novembro de 2020 a outubro de 2021.

Para estabelecer a base sobre o que seria necessário em termos de comunicação não verbal para o público supracitado, bem como, as estratégias e insumos a serem utilizados e suscitar o caráter inovador sobre o projeto, foi, inicialmente, desenvolvida uma revisão com foco em promover a imersão no tema.

Assim, foi escolhido como instrumento a ser desenvolvido, um sólido geométrico na forma de cubo, contendo em cada uma de suas seis faces uma frase curta ou palavra associada a uma imagem e cor diferentes, prezando pela harmonização e fácil leitura. As frases ou palavras representariam as principais necessidades de comunicação de pacientes em pós-operatório imediato e mediato ligados ao CCP com prejuízo vocal e dependentes de cuidados.

As frases ou palavras escolhidas para estampar o instrumento foram aquelas que, segundo a literatura científica atual, melhor se enquadravam às necessidades mais primárias e mais comuns de comunicação pelos pacientes em pós-operatório por CCP com prejuízo vocal. Assim, foram elencadas as frases curtas ou palavras: "Sim"; "Não", "Dor"; "Ir ao banheiro"; "Difícil respirar"; "Sentar/andar". A Figura 1 detalha as seis faces do cubo. 
Figura 1 - Faces do cubo.
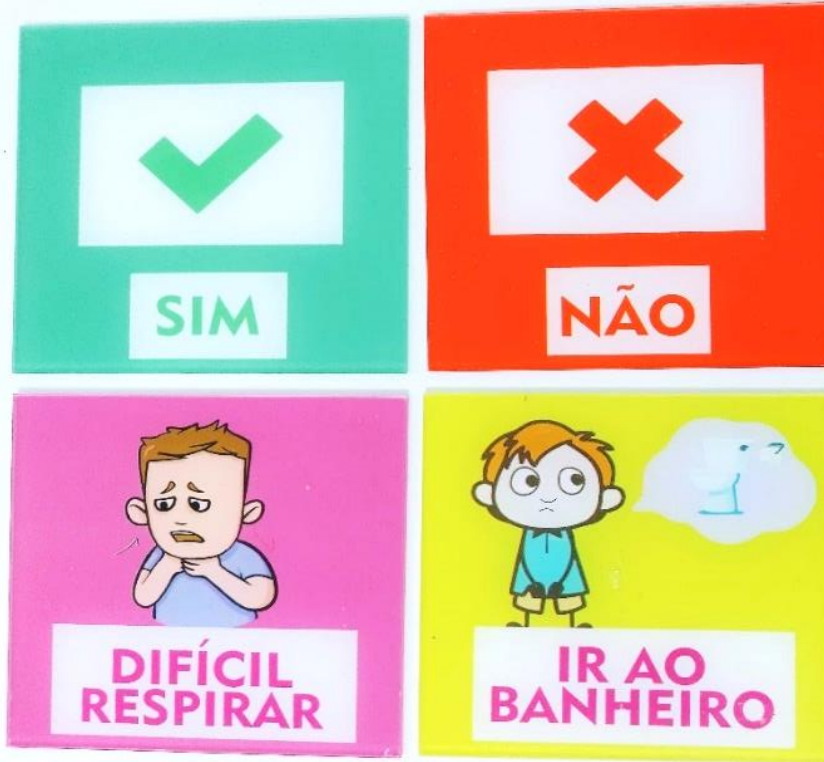

Nota: imagem construída pelos autores no software CorewDraw®.

Fonte: Figueiredo AR, et al., 2021.

Antes da construção física do instrumento, foi elaborado um esboço digital de cada face do cubo (frase ou palavra, imagem e coloração) de alta definição (720p), de forma a evitar distorções e má qualidade na impressão. O material gráfico foi elaborado, aperf eiçoado através do programa CorelDraw ${ }^{\circledR} 2018$. Esta etapa foi desenvolvida por um discente integrante do grupo de pesquisa com habilidades no manuseio do aplicativo; para formar as ilustrações que estampam o instrumento, foi utilizada como base vetores de domínio público, encontrados por meio de busca simples atrelada as frases/palavras no sítio Google Imagens ${ }^{\circledR}$ na configuração de exibição de imagens de domínio público.

Finalizada a etapa de confecção da arte digital, foi contratada uma empresa de produção gráfica para confeccionar as seis faces dos cubos, perfazendo um total de trinta e seis peça, as quais seriam posteriormente montadas. As faces foram produzidas em polimetilmetacrilato (acrílico), na fo rma de placas quadradas com dimensões de $10 \times 10 \times 0,2 \mathrm{~cm}$. A escolha da forma geométrica, do material e das dimensões visava a compatibilidade em se obter um instrumento leve, de acabamento liso e resistente, bem como, de possuir tamanho adequado a visualização e manuseio. Também foi adicionada a cada cubo uma alça de fio de polímero de poliamida (Nylon®), com $35 \mathrm{~cm}$ de comprimento e $0,2 \mathrm{~cm}$ diâmetro, fixada em um dos vértices e idealizada como forma de facilitar a manipulação e porte do instrumento.

A arte gráfica foi plotada no lado interno de cada placa (até então transparentes), sendo a escolha por decalque em vinil, garantindo alta resistência à manipulação ou atrito, também sendo um material que não desbota e suporta limpezas sucessivas, conforme aplicável e necessário no contexto em que os instrumentos foram idealizados para serem utilizados.

As etapas de montagem dos cubos foram realizadas pelos discentes integrantes do projeto de pesquisa, e os seguintes materiais usados para a composição dos cubos: placas de acrílico (seis unidades para cada cubo), fita adesiva dupla face de alta aderência de $1,2 \mathrm{~cm}$ de espessura, palitos de picolé de $11,4 \times 1 \times 0,2 \mathrm{~cm}$ (submetidos a corte), cola de madeira, cola de silicone, adesivo a base de resina epóxi e fios de Nylon® de $35 \times 0,2 \mathrm{~cm}$. Para a construção dos cubos foram necessárias os seguintes equipamentos e ferramentas: alicate de preensão e corte bico fino, lixa ํㅡㄴ 30 , tesoura sem ponta multiuso, régua milimétrica, caneta esferográfica, lápis tipo escolar.

Para obter êxito na confecção das peças, primeiramente foram montadas armações com uso dos palitos de picolé, cortados em comprimento de cerca de $8 \mathrm{~cm}$, os quais foram colados com cola de madeira formando 
peças em "L", que internamente vieram a servir de sustentação às faces acrílicas, estabelecendo a arquitetura do cada cubo. Para a montagem e aderência das placas nas referidas peças da armação se utilizou a fita adesiva dupla face. Assim, se procedeu a fixação de cada face dos cubos, uma a uma, e restando a última, antes de seu posicionamento se aplicava a alça de cada cubo, para então fechar por completo. Com a fixação da última face se encerrava a etapa de montagem, restando o acabamento. A Figura 2 e Figura 3, detalham os elementos descritos.

Figura 2 - peças da armação em "L".

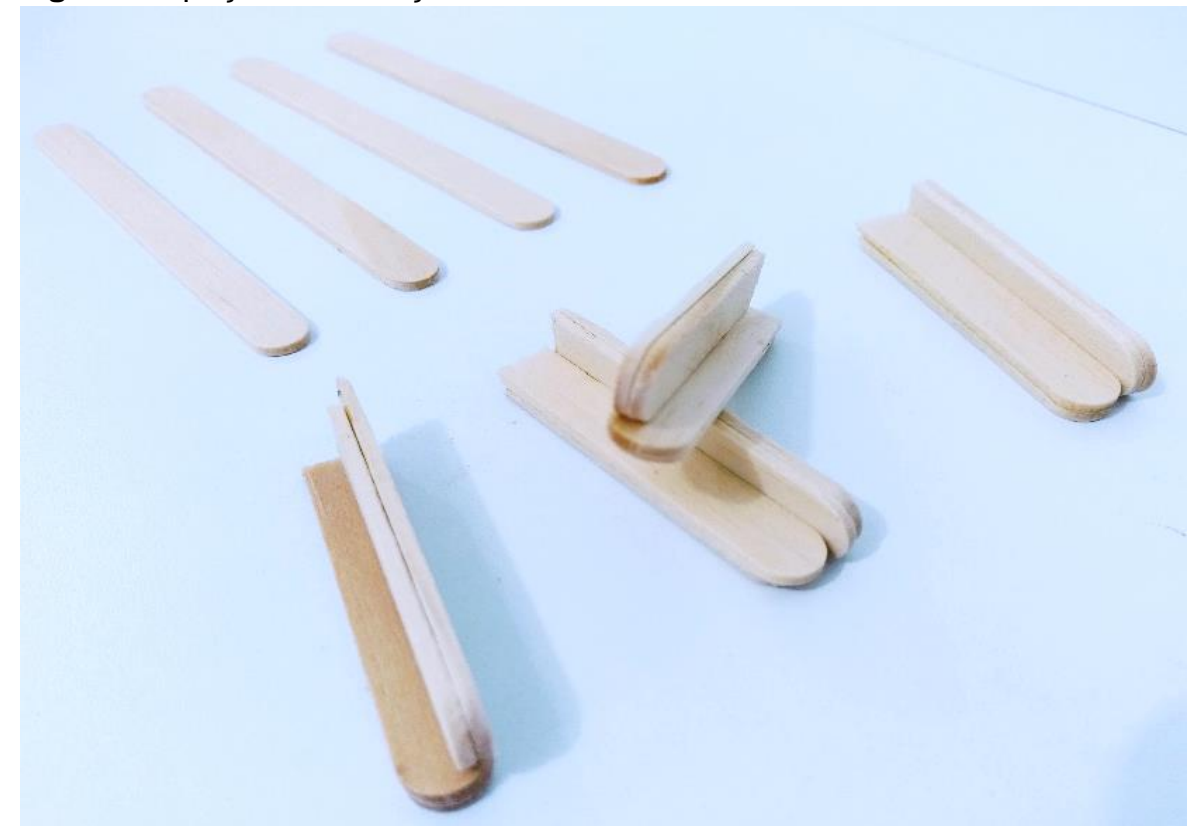

Fonte: Figueiredo AR, et al., 2021.

Figura 3 - Cubo em processo de construção

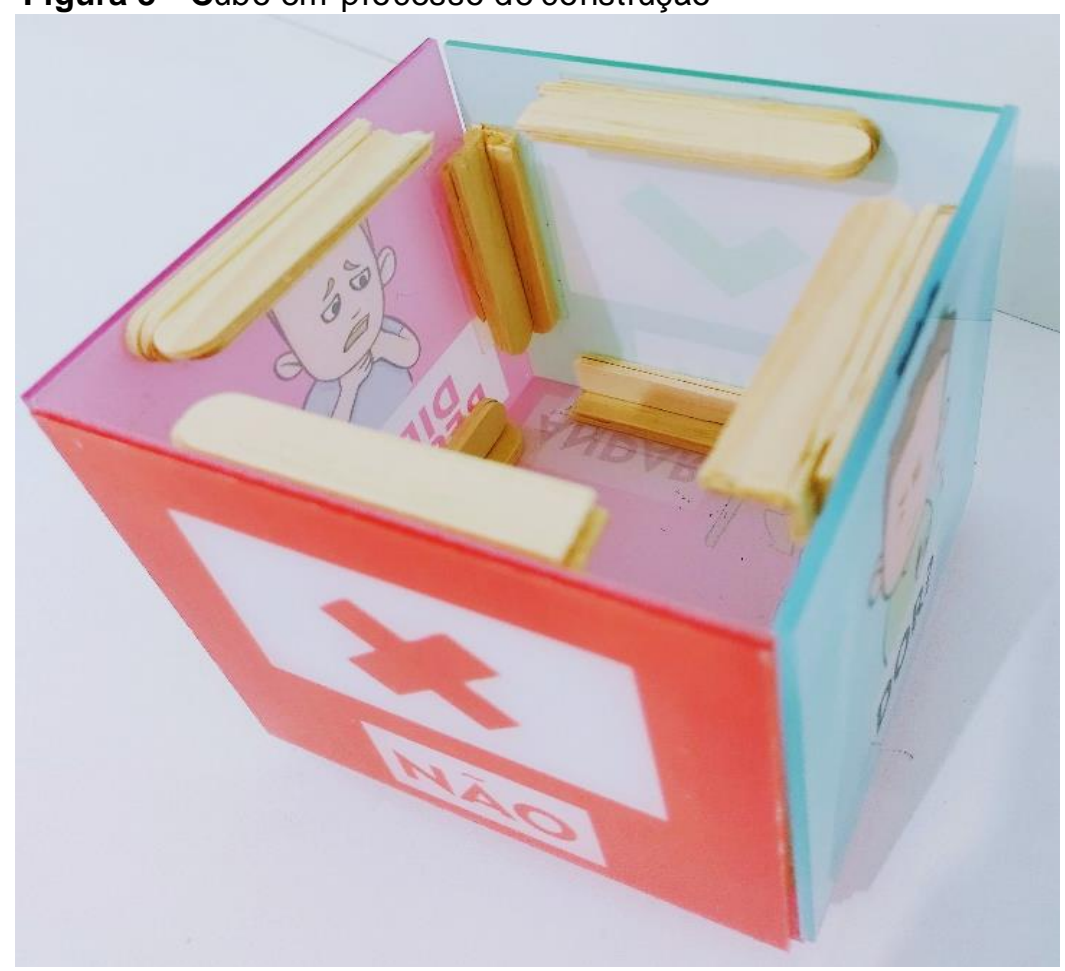

Fonte: Figueiredo AR, et al., 2021. 
Para o acabamento, cada peça foi submetida a lixamento leve de seus ângulos e arestas, removendo quaisquer eventuais asperezas, além a aplicação nas junções das placas de dois tratamentos: cola de silicone, para ef eito selante primário e, a seguir, adesivo em resina epóxi para reforço da fixação e selagem final. Novamente, após um período de doze horas, foi realizada novo lixamento e polimento para um acabamento definitivo. Foi realizada uma inspeção visual em cada cubo, buscando falhas ou pontos ainda necessitando de ajustes. Após, cada peça foi lavada em água corrente e sabão líquido, com secagem com papel toalha e mantida em ambiente arejado, consideradas então, finalizadas. A Figura 4 detalha os cubos acabados.

Figura 4 - Cubos finalizados.

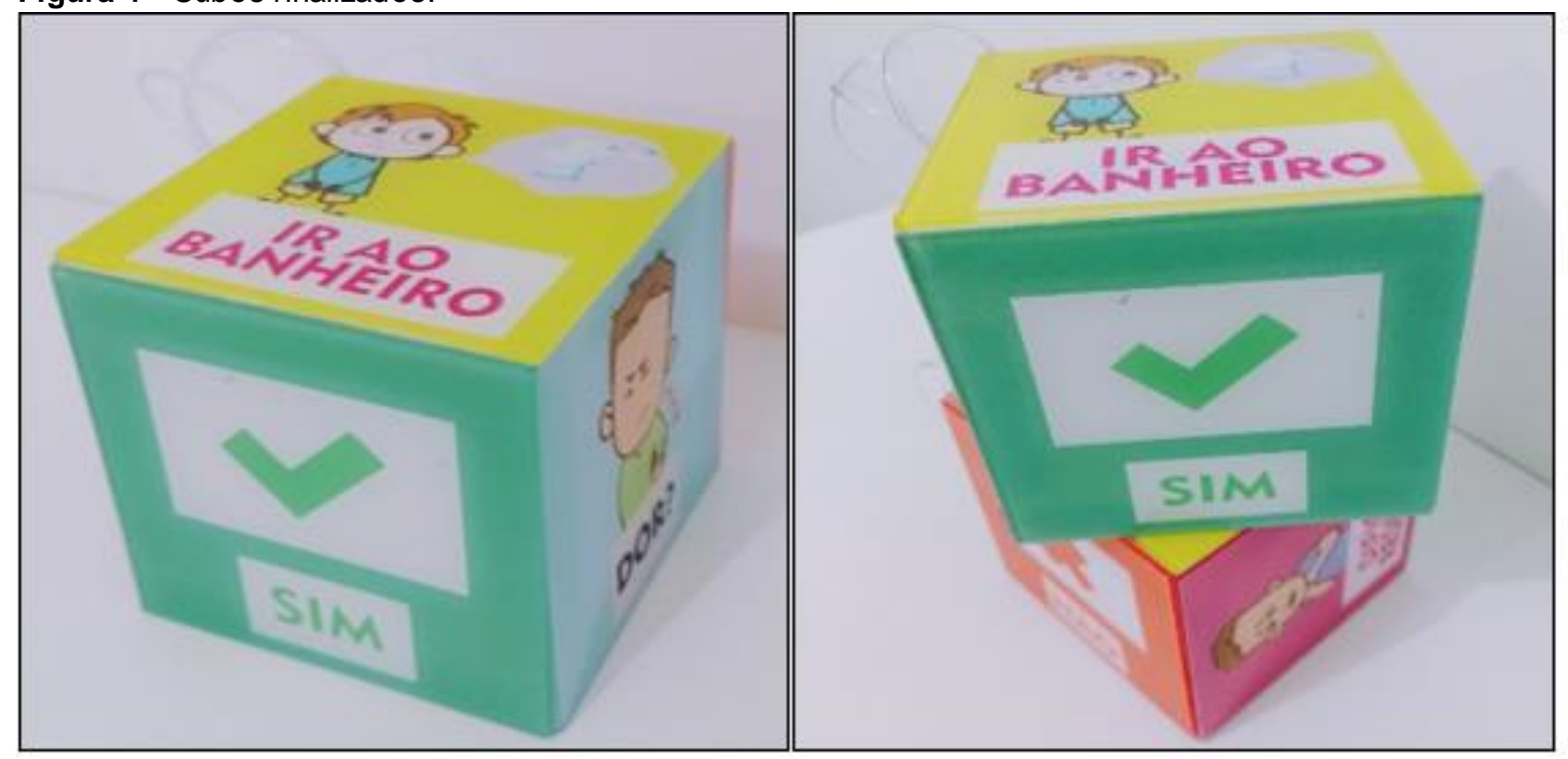

Fonte: Figueiredo AR, et al., 2021.

\section{DISCUSSÃo}

A partir da participação de acadêmicos em um programa de iniciação científica surge a oportunidade da imersão no processo de produção do conhecimento a partir de experiências que unem teoria e prática e contemplam as diversas faces do estudo científico, portanto, se beneficiando tanto com o aprendizado no desenvolvimento de atividade de pesquisa, quanto com a próprio acesso a novas práticas metodológicas e 0 contato com orientadores com expertise em determinado ramo, o que favorece a aquisição de novos conhecimentos científicos e o aprofundamento em áreas específicas de interesse. Nesse momento se descobre, aprende ou cria novos alicerces para formação, além de relacionar a teoria e a prática (ALMEIDA GLM, et al., 2018)

O acadêmico que desenvolve um projeto de iniciação científica no seio universitário adquire habilidades e conhecimentos diferenciados, voltados ao eixo da pesquisa dentro do tripé ensino, pesquisa e extensão. As atividades relacionadas à pesquisa são fundamentais para a formação acadêmica, profissional e pessoal, pois são atividades que tendem a auxiliar os estudantes a amadurecem modelos de trabalho que incluem planejamento, organização e metas com prazos, assumindo a postura de cientistas. Nesta concepção a pesquisa é caracterizada como processo educativo emancipatório e, para tanto, ela precisa ser acompanhada da capacidade de questionamento, de elaboração e reelaboração constante dapor seus construtores (LOPES MJP e SOUSA JUNIOR DL, 2018).

A pesquisa metodológica aplicada e, especialmente como nesse estudo, o desenvolvimento de uma tecnologia em saúde, contribuiu para formação dos acadêmicos de enfermagem ao promover o reconhecimento de cada etapa do método proposto, além de suscitar a percepção sobre o caráter 
multidisciplinar da prática assistencial, sobretudo pelo fato do cubo projetado atender a comunicação entre pacientes e qualquer receptor da mensagem, bem como a busca pela inovação e a criatividade, compatibilizando com o ensino/aprendizagem por metodologias ativas na formação (LIMA EIS, et al., 2017)

Nesse contexto, visualizam-se mudanças na formação do enfermeiro, associadas a um campo extenso de novidades, onde o conhecimento crítico, reflexivo e criativo é preeminente, conduzido por instrumentos tecnológicas que formam desenvolvimento e obstáculos para o ensino da enfermagem. Nesse sentido, o acadêmico ganha experiência ao desenvolver uma tecnologia em saúde dentro de sua formação e esta habilidade, que incluiu o projeto e a confecção, é levada ao decorrer de sua profissão, destacando-o no mercado de trabalho (SALVADOR PTCO, et al., 2015).

A construção de tecnologias em saúde voltadas ao aprimoramento da assistência, especificamente, uma tecnologia leve e dura, denominada como conhecimentos estruturados sob a forma de ferramentas tecnológicas, sejam materiais ou imateriais, que fomentam ou instrumentalizam a prática assistencial e ainda, podem contribuir no auxílio àquele que é cuidado ou para apoio educacional, é um meio de expandir as possibilidades de experiência dentro da formação acadêmica e, desta forma, impactar de modo positivo a atividade profissional formado, atrelando base científica e competência criativa as suas ações (ARAIS AGC, et al., 2021).

Durante o desenvolvimento de tecnologias assistivas para a saúde, os pesquisadores avaliaram estratégias para se obter linguagens e símbolos que contemplassem vocabulários coerentes com o públicoalvo, de fácil leitura, compreensão e atraentes. A utilização de ilustrações foi vantajosa ao provocar a realidade, favorece a percepção de detalhes, permitindo a idealização de processos compatíveis com realizada em que se desejava intervir (MORENO RSR, et al., 2020).

Para a construção de uma tecnologia em saúde torna-se imprescindível ser atrativo, acessível e favorável a realidade do usuário e exibir vocabulário compreensivo. Desta forma, a construção desse tipo de material ainda no ambiente acadêmico em muito favorece aptidões fundamentais para a formação do enfermeiro (MARTINS ABT, et al., 2020). A utilização de tecnologias em saúde pelo enfermeiro na prática assistencial em muito leva a resultados significativos, uma vez que haverá interrelação por meio da comunicação intermediada pelo instrumento facilitador, o que vem a favorecer a construção de vínculos e acolhimento nas atividades cotidianas dos enfermeiros (QUEIROZ PES, et al., 2017).

A partir do estudo desenvolvido em um projeto de iniciação científica foi possível construir uma tecnologia assistencial em saúde voltada a um grupo específico com prejuízo vocal. Essa construção, de uma tecnologia leve-dura, permitiu aos acadêmicos a imersão na seara da pesquisa metodológica, o que refletiu em ganhos valorosos no que se refere a aproximação entre teoria e prática, a criatividade, a capacidade de raciocínio e intervenção frente aos problemas reais, com a construção de produtos que possam ef etivamente intervir sobre problemas. Esse modelo de formação agrega habilidades ao acadêmico capazes de, mais a frente, gerar um profissional com um olhar reflexivo e com criticidade sobre a realidade, proativo e, portanto, qualificado para of erecer à sociedade um trabalho diferenciado e assertivo.

\section{REFERÊNCIAS}

1. ALCANTARA LS. Sem voz e sem vez: a mutilação no câncer de laringe e a desproteção social dos trabalhadores. Tese (Doutorado em Serviço Social). Universidade do Estado do Rio de Janeiro, Rio de Janeiro, 2017;239 p.

2. ALMEIDA GLM, et al. Iniciação cientifica por meio da pesquisa. Revista de Graduação Universidade de São Paulo (USP), 2018; 3(2): 93-97.

3. ALMEIDA JF, et al. Aconselhamento oncogenético como tecnologiaassistencialem enfermagem oncológica: revisão integrativa. Society and Development. 2021;10(2):1-8.

4. ARAIS AGC, et al. Protocolos na enfermagem: relato de experiência de uma disciplina sobre tecnologias em saúde. Revista Eletrônica Acervo Saúde, 2021;13(8): 1-7.

5. BARROS ES. Avaliação da qualidade de vida e da experiencia alimentar em pacientes submetidos ao tratam ento cirúrgico do carcinoma de células escamosas de boca. Dissertação (Mestrado em Odontologia). Pontifícia Universidade Católica de Minas Gerais, Belo Horizonte, 2017.65 p. 
6. CAÇADOR MCRC. Voz e postura. Tese (Doutorado em Medicina) - Faculdade de Ciências Médicas. Universidade NOVA de Lisboa:Portugal, 2019. 251 f.6

7. INSTITUTO NACIONAL DE CÂNCER JOSÉ ALENCAR GOMES DA SILVA (INCA). Estimativa 2020: incidência de câncer no Brasil. 2019. Disponível em: https://www.inca.gov.br/publicacoes/livros/estimativa-2020-incidencia-decancer-no-brasil. Acessado em:26 out. 2021.

8. LIMA EIS, et al. Validação de tecnologia educativa sobre primeiros socorros. Mostra interdisciplinar do curso de enfermagem, 2017;3(1):1-5.

9. LOPES MJP, JUNIOR DLS. Iniciação científica: uma análise de sua contribuição na formação acadêmica. Revista Cesumar - Ciências Humanas e Sociais Aplicadas, 2018;23(1):133-148.

10. MARTINS ABT, et al. Avaliação de uma tecnologia leve no formato de cartilha em terapia capilar. Revista Práticas Educativa, Memórias e Oralidades (PEMO), 2020;2(1): 1-17.

11. MENDES KDS, et al. Uso de gerenciador de referências bibliográficas na seleção dos estudos primários em revisão integrativa. Texto Contexto Enfermagem, 2019;28:1-13.

12. MORENO RSR, et al. Tecnologias assistivas na comunicação de paciente com deficiência auditiva em serviços de saúde do Brasil. Brazilian Journal of Development, 2020;6(8):58079-58101.

13. NEVES CPB, et al. Perfil dos pacientes com diagnóstico de câncer de cabeça de pescoço atendidos em Hospital Especializado em Recife - PE, no ano de 2014. Revista Multidisciplinar e Psicologia (online), 2017;11(37): 1-14.

14. NOGUEIRA CSF. Instrumento Cadem Adaptado: subsídio para o autocuidado de pacientes cirúrgicos por câncer de cabeça e pescoço pela enfermagem. Dissertação(Mestrado em Enfermagem). Universidade Federal do Estado do Rio de Janeiro (ÚNIRIO), Rio de Janeiro, 2017;131 f.

15. QUEIROZ PES, et al. Importância da tecnologia no processo de enfermagem para o tratamento de feridas crônicas. Revista de Enfermagem Contemporânea, 2017;6(2):158-166.

16. RODRIGUES JCQ. Tumores de laringe: o desafio terapêutico. Dissertação (Mestrado em Medicina) - Faculdade de Medicina de Lisboa. Universidade de Lisboa, Portugal, 2019;55 f.

17. RUFINO PJH. Relação da atividade física com o prognóstico pós-cirúrgico em pacientes oncológicos com cancro de cabeça e pescoço com distress. Dissertação (Mestrado em Atividade Física) - Faculdade de Desporto. Universidade do Porto, Porto, 2020; $61 \mathrm{p}$.

18. SALVADOR PTCO, et al. Tecnologia no ensino de enfermagem. Revista Baiana de Enfermagem, 2015;29(1):33-41.

19. SILVA FA, et al. Perfil epidemiológico dos pacientes com câncer de cabeça e pescoço em um Centro Oncológico no Sul do Brasil. Revista Brasileira de Cancerologia, 2020;66(1):1-8.

20. SILVA IR, et al. Conexões entre pesquisa e assistência: desafios emergentes para a ciência, a inovaçãoe a tecnologia na enfermagem. Texto \& Contexto de Enfermagem, 2017;26(4):10-15. 